\title{
A THEOREM ON FINITE GENERATION OF A RING
}

\author{
MASAYOSHI NAGATA \\ Dedicated to late Professor TAdasi Nakayama
}

The fourteenth problem of Hilbert asked finite generation of a certain class of rings and had a counter-example (cf. [4]). On the other hand, many mathematicians gave various sufficient conditions for finite generation of such rings (see, for instance, [9], [5] and [8]). The purpose of the present paper is to give a new sufficient condition. The class of rings to be treated is much more general than those treated before, except for the one in [8].

As for the terminology, we use mainly the one used in our book [7], hence a ring means a commutative ring with identity and a local ring means a Noetherian local ring. When $B$ is a ring, we shall understand by a $B$-algebra a ring which is a $B$-module, and by a $B$-algebra of finite type a $B$-algebra which is finitely generated over the natural homomorpic image of $B$.

Our main result can be stated as follows ${ }^{11}$ :

MaIn Theorem. Let $B$ be a pseudo-geometric ring and let $A$ be a B-algebra of finite type. For a $B$-subalgebra $R$ of $A, R$ modulo its radical is a $B$-algebra of finite type if $R$ is strongly submersive in $A$.

Here, the strong submersiveness is defined as follows:

We say that a ring $R$ is strongly submersive ${ }^{2)}$ in a ring $A$ if (i) $A$ is an $R$-algebra and (ii) it holds that if $\mathfrak{p}$ is a prime ideal of height 0 in $R$ and if $V$ is a valuation ring of the field of quotients $K(\mathfrak{p})$ of $R / p$ such that $V \supseteq R / \mathfrak{p}$, then, there is a pair of a valuation ring $V^{\prime}$ (of a field containing $K(p)$ ) which dominates $V$ and a homomorphism $\phi$ from $A$ in $V^{\prime}$ such that the natural homomorphism

Received May 24, 1965.

1) The case where $B$ is a field or a pseudo-geometric Dedekind domain was established by the writer while he was staying at Woods Hole, Mass. in order to attend 1954 A.M.S. Summer Institute on Algebraic Geometry. The writer likes to express his thanks to Dr. David Mumford for the discussion on the condition to be supposed.

2) The term "strongly submersive" is introduced because our condition implies universal submersiveness in the sense of Grothendieck [2], Exposé 9. 
from $R$ into $V^{\prime}$ through $V$ coincides with the one through $A \stackrel{\phi}{\longrightarrow} V^{\prime}$.

\section{$\S 1$. Some preliminary results on valuation rings}

Lemma 1.1. Let $K$ and $K^{\prime}$ be fields such that $K \subseteq K^{\prime}$ and let $x$ be a transcendental element over $K^{\prime}$. If valuation rings $V^{\prime}$ and $V^{\prime \prime}$ of $K^{\prime}$ and $K(x)$ respectively are given so that they dominate a valuation ring $V$ of $K$ in common, then there is a valuation ring $V^{*}$ of $K^{\prime}(x)$ such that $V^{*}$ dominates both $V^{\prime}$ and $V^{\prime \prime}$.

Proof. If the assertion is proved for a larger $K^{\prime}$ then the assertion for the original $K^{\prime}$ follows from it. Therefore we may assume that $K^{\prime}$ is algebraically closed. Let $\bar{K}$ be the algebraic closure of $K$ (in $K^{\prime}$ ) and set $\bar{V}=V^{\prime} \cap \bar{K}$. Then a valuation ring of $\bar{K}$ dominates $V$ if and only if it is a conjugate of $\bar{V}$ over $K$. We take a valuation ring $\bar{V}^{\prime \prime}$ of $\bar{K}(x)$ which dominates $V^{\prime \prime}$, then $\bar{V}^{\prime \prime} \cap \bar{K}$ is a valuation ring dominating $V$, whence there is an automorphism $\sigma$ of $\bar{K}$ over $K$ such that $\left(\bar{V}^{\prime \prime} \cap \bar{K}\right)^{\sigma}=\vec{V}$. We extend $\sigma$ to an automorphism of $\bar{K}(x)$ over $K(x)$; denote the extension by the same $\sigma$. Then $\bar{V}^{\prime \prime}$ dominates $\bar{V}$. Obviously, $\bar{V}^{\prime \prime \prime}$ dominates $V^{\prime \prime}$. Therefore, we have only to prove the assertion under the additional assumption that $K=\bar{K}$. Since $K(x)=K\left(x^{-1}\right)$, we may assume that $x \in V^{\prime \prime}$. Then, considering $x-c$ with $c \in V$ if necessary, we may assume that $x$ is a unit in $V^{\prime \prime}$. We denote by $v, v^{\prime}$ and $v^{\prime \prime}$ valuations whose rings are $V, V^{\prime}$ and $V^{\prime \prime}$ respectively such that $v^{\prime}$ and $v^{\prime \prime}$ are extensions of $v$. (1) If there is no element $c$ in $V$ such that $V^{\prime \prime}(x-c)>0$, then $V^{\prime \prime}=V(x)$ and therefore it is enough to take $V^{*}$ to be $V^{\prime}(x)$. (2) Assume that there is an element $c$ of $V$ such that $v^{\prime \prime}(x-c)>0$ and such that $\alpha>v^{\prime \prime}(x-c)>\beta(\alpha, \beta \in$ value group of $v)$ implies $\alpha>v^{\prime \prime}\left((x-c)^{n}\right)>\beta$ for every natural number $n$. Then for each polynomial $f(x) \in K[x], v^{\prime \prime}(f(x))$ is defined by the minimum of $\left.v^{(} c_{i}\right)+i \cdot v^{\prime \prime}(x-c)$ where $f(x)=\sum_{i} c_{i}(x-c)^{i}\left(c_{\imath} \in K\right)$. Therefore we see easily the existence of a valuation $v^{*}$ of $K^{\prime}(x)$ which is an extension of both $v^{\prime}$ and $v^{\prime \prime}$. (3) Consider the remaining case. Let $M$ be the set of all pairs $(c, d)$ of elements of $V$ such that $v\left(d^{2}\right)>v^{\prime \prime}(x-c) \geq v(d)>0$. Since the cases (1) and (2) are excluded and since $K$ is algebraically closed, the set $M$ is not empty. Set $D=\cap_{(c, d) \in M} d V^{\prime}$. $(3, \mathrm{i})$ Assume that there is an $a \in V^{\prime}$ such that $a-c \in d V^{\prime}$ for all $(c, d) \in M$. $(3, \mathrm{i}, \uparrow)$ If, furthermore $D$ is a non-zero principal ideal $e V^{\prime}$, then we $\epsilon$ xtend $v^{\prime}$ to a valuation $v^{*}$ of $K^{\prime}(x)$ by $v^{*}\left(\sum a_{i}(x-a)^{i}\right)=\min v^{\prime}\left(a_{i}\right)+i \cdot v^{\prime}(e)$, and this 
valuation is an extension of $v^{\prime \prime}$, and therefore the valuation ring $V^{*}$ of $v^{*}$ is a required ring in this case. $(3, \mathrm{j}, \square)$ Assume that $D$ is not a principal ideal. Let $G^{\prime}$ be the value group of $v^{\prime}$. Introducing a new element $\delta$, we construct the direct sum $G^{*}$ of $G$ and the free additive group generated by $\delta$. A linear order in $G^{*}$ is derived from the definition that $\alpha+m \delta \geq \beta+n \delta(\alpha, \beta \in G ; m, n$ non-negative integers) if and only if for every $d$ such that $(c, d) \in M$, there is a $\left(c^{\prime}, d^{\prime}\right) \in M$ such that $\alpha+m \cdot v\left(a^{\prime}\right) \geq \beta+n \cdot v(d)$. Then we extend $v^{\prime}$ to a valuation $v^{*}$ of $K^{\prime}(x)$ by that $v^{*}\left(\sum a_{i}(x-a)^{i}\right)=\min v^{\prime}\left(a_{i}\right)+i \cdot \delta$. Then the valuation ring $V^{*}$ of $v^{*}$ is the required ring in this case. $(3, i,>)$ Assume now that $D=0$. Then we consider $G^{*}$ as above, but its linear order being defined by that $\delta$ is larger than any element of $G$, and we have required ring similarly. (3 ii) We have now the case where there is no $a$ such that $a-c \in d V^{\prime}$ for all $(c, d) \in M$. Then, for each $a \in K$, there is a $(c . d) \in M$ such that $a-c \notin d V^{\prime}$. Let us denote by $M(a)$, the set of such $(c, d)$. When a polynomial $f(x) \in K^{\prime}[x]$ is given, we consider all zero-points $a_{1}, \ldots a_{r}$ of $f(x)$. For each $a_{i}$, we take an element $\left(c_{i}, d_{i}\right)$ of $M\left(a_{i}\right)$ and let $(c, d)$ be an element of $M$ such that $d \in d_{i} V$ for every $i$. We define $v^{*}(f(x))$ to be $v^{\prime}(f(c))$. We shall prove now that this definition is independent of the particular choice of $(c, d) \in M$. Indeed, assume that $\left(c^{*}, d^{*}\right) \in M$ and that $d^{*} \in d V . f(x)=b \prod_{i=1}^{n}\left(x-a_{i}\right)$, whence $f(c)=b \Pi_{i}\left(c-a_{i}\right)$, $f\left(c^{*}\right)=b \Pi_{i}\left(c^{*}-a_{i}\right)$. Since $x-c \in d V^{\prime \prime}$ and $x-c^{*} \in d^{*} V^{\prime \prime} \subseteq d V^{\prime \prime}$, we see that $c-c^{*} \in d V$. Therefore $f(c)-f\left(c^{*}\right) \in b d^{n} V^{\prime}$, Since $(c, d) \in M\left(a_{i}\right)$, we have $v^{\prime}\left(c-a_{i}\right)<v(d)$ and therefore we see that $v^{\prime}(f(c))=v^{\prime}\left(f\left(c^{*}\right)\right)$. From this, it follows easily that $v^{*}$ defines a valuation of $K^{\prime}(x)$ which is an extension of both $v^{\prime}$ and $v^{\prime \prime}$. Therefore the valuation ring $V^{*}$ of $v^{*}$ is the required ring in this case. Thus the proof of Lemma 1 is completed.

LEMMA 1.2. If a ring $R$ is strongly submersive in a ring $A$, and if $a$ is an ideal of $R$, then $R / a$ is strongly submersive in $A / a A$.

Proof. Let $\bar{n}$ be a prime ideal of height 0 in $R / \mathfrak{a}$ and let $\phi$ be the natural homomorphism from $R$ into $R / \mathfrak{a}$. Let $p$ be $\phi^{-1}(\bar{p})$ and let $\mathfrak{q}$ be a prime ideal of $R$ of height 0 which is contained in p. Let $K(\mathfrak{p})$ and $K(\mathfrak{q})$ be the fields of quotients of $R / \mathfrak{p}=(R / \mathfrak{a}) / \bar{p}$ and $R / \mathfrak{q}$ respectively. Let $\bar{V}$ be a valuation ring of $K(p)$ which contains $R / p$. We take a valuation ring $V_{0}$ of $K(q)$ which dominates $R_{\mathfrak{p}} / \mathfrak{q} R_{\mathfrak{p}}$. The residue class field $K\left(V_{0}\right)$ of $V_{0}$ contains $K(\mathfrak{p})$. Let $\bar{V}^{\prime}$ be a valuation ring of $K\left(V_{0}\right)$ such that it dominates $\bar{V}$ and we take the composite 
$V$ of $V_{0}$ with $\bar{V}^{\prime}$. Then $V$ is a valuation ring of $K(\mathfrak{q})$ which contains $R / \mathfrak{q}$. By the condition that $R$ is strongly submersive, the natural homomorphism $\sigma$ from $R$ into $V$ can be extended to a homomorphism from $A$ into a valuation ring $V^{*}$ which dominates $V$. Taking the minimal prime divisor $m$ of $\mathrm{m}_{0} V^{*}$ $\left(\mathrm{m}_{0}=\right.$ the maximal ideal of $\left.V_{0}\right)$, we see that $V^{*} / \mathrm{m}$ is a valuation ring dominating $\bar{V}$. The natural homomorphism from $A$ into $V^{*} / \mathrm{m}$ induces a natural homomorphism from $A / \mathrm{o} A$ into $V^{*} / \mathrm{m}$ and the homomorphism is an extension of the natural homomorphism from $R / 0$ into $\vec{V}$. Thus the lemma is proved completely.

As a corollary to these two lemmas, we have

THEOREM 1. Let $R$ be a ring and let $A$ be an $R$-algebra. If $R$ is strongly submersive in $A$, then for an arbitrary R-algebra $R^{\prime}, R^{\prime}$ is strongly submersive in $A \otimes{ }_{R} R^{\prime}$.

Lemma 1.3. Let $K$ be a normal quasi-local ring with field of quotients $K$ and let $L$ be an algebraic extension field of $K$. Let $R^{*}$ be the integral closure of $R$ in $L$ and let $\mathrm{m}^{*}$ be a maximal ideal of $R^{*}$. Then $R=R^{*} \mathrm{~m}^{*} \cap K$.

Proof. We may assume that $L$ is a normal extension of $K$. If $V$ is a valuation ring of $K$ which dominates $R$, then there is a valuation ring $V^{*}$ of $L$ such that $V^{*} \cap K=V . \quad V^{*}$ contains $R^{*}$ and the maximal ideal of $V^{*}$ lies over some maximal ideal of $R^{*}$. Therefore there is an automorphism $\sigma$ of $L$ over $K$ such that the maximal ideal of $V^{* \sigma}$ lies over $\mathrm{m}^{*}$. Then $V^{* \sigma}$ contains $R^{*} \mathrm{~m}$, whence $V=V^{* \sigma} \cap K$ contains $R^{*} \mathfrak{m} \cdot \cap K$. Since the intersection of all such $V$ is $R$. we see that $R$ contains $R^{*}{ }^{*} \cap K$, and we see the assertion.

Now we are to prove

Theorem 2. Let $B$ be a Noetherian integral domain. Assume that a valuation ring $V$ of a field $K$ is the derived normal ring of a locality $P$ over $B$. Then for an arbitrary subfield $K^{\prime}$ of $K$ containing $B$, the valuation ring $V^{\prime}=V \cap K^{\prime}$ is the derived normal ring of a suitable locality over $B$.

Proof. We may assume that $B$ is a local ring dominated by $V$ and has $K^{\prime}$ as its field of quotients. Let $A$ be an affine ring over $B$ such that $P=A_{\Re}$ with a prime ideal $\mathfrak{B}$ of $A$ and let $a_{1}, \ldots, a_{n}$ be a set of generators of $A$ over $B$. Let $m$ and $\mathrm{m}^{\prime}$ be the maximal ideals of $V$ and $V^{\prime}$ respectively.

(1) When $K$ is algebraic over $K^{\prime} . \quad V$ is the ring of quotients of the integral closure $V^{*}$ of $V^{\prime}$ in $K$ with respect to $\mathrm{m} \cap V^{*}$. Each $a_{i}$ is written in the form 
$b_{i} / c_{i}$ with $b_{i}, c_{i}$ in $V^{\prime}$ but $c_{i}$ not in $\mathrm{m} \cap V^{*}$. Therefore, considering all of $b_{i}, c_{i}$ instead of the $a_{i}$, we may assume that all the $a_{i}$ are integral over $V^{\prime}$. Let $f_{i}(x)$ be a monic polynomial over $V^{\prime}$ which has $a_{i}$ as a root for each $i$. Then, enlarging $B$, we may assume that $B$ contains all of the coefficients of these $f_{i}(x)$ and that the derived normal ring $B^{*}$ of $B$ is quasi-local. Since $A$ is integral over $B^{*}, V$ is a ring of quotients of the integral closure $B^{* *}$ of $B^{*}$ in $K$ with respect to $m \cap B^{* *}$. Therefore $B^{*}=V^{\prime}$ by Lemma 1.3. Thus this case is settled.

(2) The proof of the other case will be done using induction on the transcendence degree of $K$ over $K^{\prime}$. Let $x$ be an element of $V$ which is transcendental over $V^{\prime}$. Then, by the induction assumption, $V \cap K^{\prime}(x)$ is the derived normal ring of a locality over $B$. Therefore, we may assume that $K=K^{\prime}(x)$. Since $V$ is the derived normal ring of a locality over the Noetherian valuation ring $V^{\prime}$, the residue class field $V / \mathrm{m}$ is not algebraic over $V^{\prime} / \mathrm{m}^{\prime}$. Hence, in choosing $x$ above, we may do so that $x$ modulo $m$ is transcendental over $V^{\prime} / \mathrm{m}^{\prime}$. Then (under this new $K=K^{\prime}(x)$ ), we have $V=V^{\prime}(x)$. Therefore. as in the case (1) above, we may assume that $a_{i}$ are all polynomials in $x$ with coeffieients in $V^{\prime}$ and also that $a_{1}=x$. Then, enlarging $B$, we may assume that $B$ contains all of the coefficients of the polynomials $a_{i}$ and also that the derived normal ring $B^{*}$ of $B$ is quasi-local. Then, since $V$ is a ring of quotients of the derived normal ring of the integral closure $B^{*}[x]$ of $B[x]$ in $K=K^{\prime}(x)$, we see easily that $B^{*}=V^{\prime}$. Thus the proof of our theorem is completed.

\section{§ 2. An ideal-transform}

We shall expose here some facts on an ideal-transform introduced in [3]. Let $a$ be an ideal of a ring $R$ and let $Q$ be the total quotient ring of $R$. We denote by $\mathfrak{a}^{-n}$ the set of elements $b$ of $Q$ such that $b a^{n} \subseteq R$. Since $a^{-n} \mathfrak{a}^{-m} \subseteq \mathfrak{a}^{-(m+n)}$, the union $S$ of all $a^{-n}(n=1,2, \ldots)$ forms a subring of $Q$ containing $R$. This $S$ is called the a-transform of $R$ and is denoted by. $T(a)$. This is the set of elements $z$ of $Q$ such that $z a^{n} \subseteq R$ for some $n$. Therefore we have

LEMMA 2.1. Let $\mathfrak{a}$ and $\mathfrak{b}$ be ideals of a ring $R$. If $\mathfrak{a}^{n} \subseteq \mathfrak{b}$ for some natural number $n$, then $T(\mathfrak{b})$ is contained in $T(\mathfrak{a})$. Consequently, if $\mathfrak{a}^{n} \subseteq \mathfrak{b}$ and $\mathfrak{b}^{m} \subseteq \mathfrak{a}$ for some natural numbers $n$ and $m$, then $T(\mathfrak{a})=T(\mathfrak{b})$. As a particular case, if these ideals $\mathfrak{a}$ and $\mathfrak{b}$ have the same radical and if both $\mathfrak{a}$ and $\mathfrak{b}$ have finite basis, then $T(\mathfrak{a})=T(\mathfrak{b})$. 
Lemma 2.2. Let a be an ideal of a ring $R$. Assume that a contains a nonzero-divisor a. Then $\mathfrak{a}^{-n}$ is the set of $b / a^{n}$ with $b \in a^{n} R: \mathfrak{a}^{n}$.

Proof. $b \in a^{n} R: a^{n}$ if and only if $b a^{n} \subseteq a^{n} R$, and the assertion is easy.

Lemma 2.3. Let a and $R$ be as Lemma 2.2 above. Let $R^{\prime}$ be a ring such that $R \subseteq R^{\prime} \subseteq T(a)$. Then there is a one to one correspondence between the set of prime ideals $\mathfrak{p}$ of $R$ not containing a and the set of prime ideals $\mathfrak{p}^{\prime}$ of $R^{\prime}$ not containing $a R^{\prime}$ in such a way that if $\mathfrak{p}$ corresponds to $\mathfrak{p}^{\prime}$ then $\mathfrak{p}=\mathfrak{p}^{\prime} \cap R$. In this case, $R_{\mathfrak{p}}=R^{\prime}$ '.

Proof. Let $\mathfrak{p}$ be a prime ideal of $R$ which does not contain $\mathfrak{a}$. Then there is non-zero-divisor $a$ in $a$ which is not in $p$. Then $R^{\prime} \subseteq T(a) \subseteq R\left[a^{-1}\right]$ and $p R\left[a^{-1}\right]$ is prime, whence $\mathfrak{p}^{\prime}=\mathfrak{p} R\left[a^{-1}\right] \cap R^{\prime}$ is a prime ideal of $R^{\prime}$ and $\mathfrak{p}^{\prime} \cap R=\mathfrak{p} R\left[a^{-1}\right] \cap R$ $=\mathfrak{p}, R_{\mathfrak{p}^{\prime}}^{\prime}=R\left[a^{-1}\right]_{\mathfrak{p k}\left[a^{-1}\right]}=R_{\mathfrak{p}}$. Conversely, if $\mathfrak{p}^{\prime}$ is a prime ideal of $R^{\prime}$ which does not contain $\mathfrak{a} R^{\prime}$, then $\mathfrak{p}=\mathfrak{p}^{\prime} \cap R$ does not contain $\mathfrak{a}$ and by the above observation, we have $R_{\mathfrak{p}^{\prime}}^{\prime}=R_{\mathfrak{p}}$. Thus the assertion is proved completely.

Lemma 2.4. Let a be non-zero ideal of Krull ring $R$. Then $T(a)$ is the intersection of all $R_{\mathfrak{p}}$, letting $\mathfrak{p}$ run through all prime ideals of height 1 which do not contain a. Hence $T(a)$ is again a Krull ring.

Proof. $T(\mathfrak{a}) \subseteq R_{\mathfrak{p}}$ by Lemma 3 , hence $T(\mathfrak{a})$ is contained in the intersection $D$ of all such $R_{\mathfrak{p}}$. Let $\mathfrak{q}_{1}, \ldots, \mathfrak{q}_{s}$ be all of prime ideals of height 1 which contain a. Let $d$ be an element of $D$. Since $R_{\mathfrak{q}_{i}}$ is a Noetherian valuation ring for each $i$, there is a natural number $n$ such that $d \mathfrak{a}^{n} \subseteq R_{\mathfrak{q}_{i}}$ for each $i$. By the finiteness of the number of $q_{i}$, we can take $n$ to be common for all $i$. Since $d \in D$, we have then $d \mathfrak{a}^{n} \subseteq R$, and we have $d \in T(a)$. Thus $D=T(\mathfrak{a})$.

REMARK. In the above situation, $T(a)$ is determined by the set of prime ideals $q_{1}, \ldots, q_{s}$ of height 1 which contain $a$.

From this remark, it follows immediately the following

COROLlaRY. In the above situation, there are two elements $a_{1}, a_{2}$ of a such that $T(\mathfrak{a})=T\left(a_{1} R+a_{2} R\right)$.

Lemma 2.5. Assume that an ideal a of a ring $R$ has a finite basis. Then for an arbitrary ring $R^{\prime}$ between $R$ and $T(a)$, we have $T(a)=T\left(a R^{\prime}\right)$.

Proof. In general, since $R \subseteq R^{\prime}$, we have $T(a) \subseteq T\left(a R^{\prime}\right)$, even if a have no 
finite basis. Let $b$ be an element of $T\left(a R^{\prime}\right)$. Then $b a^{n} R^{\prime} \subseteq R^{\prime}$ for a ratural number $n$. Let $a_{1}, \ldots, a_{s}$ be a basis for $a^{n}$. Then we have $b a_{i} \in R^{\prime} T(a)$. Thefore there is a natural number $m$ such that $b a_{i} a^{m} \subseteq R$ for all $i$. Then we have $b a^{n+m} \subseteq R$, and $b \in T(a)$. Thus $T(a)=T\left(a R^{\prime}\right)$.

Corollary. Let a be an ideal of a Krull ring $R$. Then a $T(a)$ is not of height 1.

Lemma 2.6. Let $B$ be a ring ani let a be an ideal of a B-algebra $R$. Assume that a contains a non-zero-divisor $a$ and let $B^{*}$ be another $B$-algebra such that $\otimes_{B} B^{*}$ is exact (i.e., $B^{*}$ is a ring which is a flat $B$-module). Then $a^{-n}\left(R \otimes_{B} B^{*}\right)$ $=\left(\mathfrak{a}\left(R \otimes B^{*}\right)\right)^{-n}$ and therefore $T\left(\mathfrak{a}\left(R \otimes B^{*}\right)\right)=T(\mathfrak{a}) \otimes B^{*}$.

Proof. Denoting by $\phi$ the natural homorphism from $R$ into $R \otimes B^{*}$, we see that $\left(a\left(R \otimes B^{*}\right)\right)^{-n}=\left\{b^{*} / \phi(a)^{n} \mid b^{*} \in a^{n}\left(R \otimes B^{*}\right): a^{n}\left(R \otimes B^{*}\right)\right\}=\left\{b^{*} / \phi(a)^{n} \mid b^{*} \in\right.$ $\left.\left(a^{n} R: a^{n}\right) \otimes B^{*}\right\}=a^{-n} \otimes B^{*}$. Thus the assertion is proved.

REMARK. Observe that Lemma 2.6 can be applied when $B^{*}$ is a ring of quotients of a polynomial ring over $B$, hence in particular when $B^{*}$ is either a ring of quotients of $B$ or $B^{*}=B(x)$ with a transcendental element $x$ over $B$.

Let $a$ be an ideal of a ring $R$. We say that the a-transform is finite if there is a natural number $n$ such that $T(a)$ is generated by $a^{-n}$. When $R$ is Noetherian and a contains a non-zero-divisor, this condition is equivalent to that $T(a)$ is finitely generated over $R$.

Lemma 2.7. Let a be an ideal of a pseudo-geometric normal ring $B$. Then the following three conditions are equivalent to each other.

(1) The a-transform of $B$ is finite.

(2) For every maximal ideal $\mathfrak{n}$ of $B$, the a $B_{\mathfrak{m}}$-transform of $B_{\mathfrak{m}}$ is finite.

(3) For every prime ideal $\mathfrak{p}$ of $B$, the $\mathfrak{a} B_{\mathfrak{p}}$-transform of $B_{\mathfrak{p}}$ is finite.

Pronf. Lemma 2.6 shows that (1) implies (2) and that (2) implies (3). Therefore we have only to show that (3) implies (1). Assume that (1) does not hold. Starting with $B=B_{0}$, we define a sequence of rings $B_{0}, B_{1}, \ldots$ as follows: When $B_{i}$ is defined, we define $B_{i+1}$ to be the derived normal ring of one $B\left[a^{-n}\right]$ which contains $\left(a B_{i}\right)^{-1}$. Then this sequence is well defined and $T(a)$ is the union of all the $B_{i}$, because (i) $T(a)$ is a Krull ring by Lemma 2.4, hence is a normal ring, which implies that the derived normal ring of any 
$B\left[a^{-m}\right]$ is contained in $T(a)$, (ii) since $B$ is pseudo-geometric and since $B\left[a^{-n}\right]$ is finitely generated over $B, B\left[a^{-n}\right]$ is also pseudo-geometric, hence the derived normal ring of $B\left[a^{-n}\right]$ is finitely generated over $B$, (iii) therefore $B_{i}\left[\left(a B_{i}\right)^{-1}\right]$ is finitely generated over $B$ and (iv) Lemma 2.5 shows that $B_{i}\left[\left(a B_{i}\right)^{-1}\right]$ is contained in $T(a)$ hence the finite generation of it shows that there is one $n$ such that this ring is contained in $B\left[a^{-n}\right]$. If there exists one $i$ such that $a B_{i}$ is not of height 1 , then $T(a)=B_{i}$ by Lemma 2.4. This is not the case. Let $a_{i}$ be the intersection of prime divisors of height 1 of $a B_{i}$. Then $a_{0} \subseteq a_{1} \subseteq \cdots$ $\subseteq a_{i} \subseteq a_{i+1} \subseteq \cdots$ and the union $a^{*}$ of all the $a_{i}$ is an ideal of $T(a)$. $a^{*}$ does not contain 1 because no $a_{i}$ contains 1 . Let $\mathfrak{p}^{*}$ be a prime ideal of $T(a)$ such that $\mathfrak{p}^{*}$ contains $a^{*}$ and set $\mathfrak{p}=\mathfrak{p}^{*} \cap B$. Then we see that the $a B_{\mathfrak{p}}$-transform of $B_{\mathfrak{p}}$ is not finite. Thus (3) implies (1), and the proof of Lemma 2.7 is completed.

Lemma 2.8. Let a be an ideal of an integral domain $R$. If $b \in R$ generates $a$ prime ideal in $R$ and if $a \equiv b R$, then $b$ generates a prime ideal in $T(a)$.

Proof. Let $a$ be a non-zero element of $a$ which is not in $b R$. Then an arbitrary element of $T(a)$ is of the form $q / a^{n}$ with $q \in a^{n} R: a^{n}$ by Lemma 2.2. Assume that $\left(q / a^{n}\right)\left(q^{\prime} / a^{m}\right) \in b T(\mathfrak{a}) \quad\left(q \in a^{n} R: a^{n}, q^{\prime} \in a^{m} R: \mathfrak{a}^{m}\right)$. This shows that $q q^{\prime} / a^{n_{+}+m}=b q^{\prime \prime} / a^{t}$ with $q^{\prime \prime} \in a^{t} R: a^{t}$. Then, replacing one of $n, m, t$ by a larger number if necessary, we may assume that $t=n+m$. Then we have $q q^{\prime}=b q^{\prime \prime}$. Since $b R$ is a prime ideal, one of $q, q^{\prime}$, say $q$ is in $b R$, and $q=q^{*} b$ with $q^{*} \in R$. Since $\left(a^{n} R: a^{n}\right): b R=a^{n} R: b a^{n}=\left(a^{n} R: b R\right): a^{n}=a^{n} R: a^{n}$, we see that $q^{*} / a^{n} \in T(a)$, and we see that $q / a^{n} \in b T(a)$. Thus $b$ is a prime element in $T(\mathfrak{a})$.

Lemma 2.9. Let q be a prime ideal of a ring $R$ and let a be an ideal of $R$ which contains a non-zero-divisor and such that $a$ qq. Let $\mathrm{q}^{\prime}$ be the prime ideal of $T(a)$ which lies over $q$ and consider the derived normal rings $R^{*}$ and $R^{*}$ of $R / \mathfrak{q}$ and $T(\mathfrak{a}) / q^{\prime}$ respectively. If $R^{*}$ and $R^{*}$ are Krull rings and if height $a R^{\prime *} \geq 2$, then $R^{*}$ is the $a R^{*}$-transform $T\left(a R^{*}\right)$ of the ring $R^{*}$.

Proof. It is obvious by definition that $T\left(a R^{*}\right)$ contains $T(a) / q^{\prime}$. Since $T\left(a R^{*}\right)$ is a Krull ring hence is normal, we see that $T\left(\mathfrak{a} R^{*}\right)$ contains $R^{* *}$. Therefore we have, by the corollary to Lemma 2.4 and by Lemma 2.5 , that $T\left(a R^{*}\right)=T\left(a R^{*}\right)=R^{*}$. Thus the lemma is proved.

REMARK. If we do not assume that $R^{* *}$ is a Krull ring and height $a R^{\prime} \geq 2$, 
then we have that $T\left(a R^{*}\right)=T\left(a R^{*}\right)$.

\section{$\S 3$. Proof of the main theorem}

We begin with a well known lemma :

Lemma 3.1. Let $B$ be a Noetherian ring and let $A$ be a B-algebra of finite type. If $A$ is integral over its $B$-subalgebra $R$, then $R$ is also a B-algebra of finite type.

Proof. We may assume that $R$ contains $B$ Let $a_{1}, \ldots, a_{n}$ be a set of generators of $A$ over $B$. For each $a_{i}$, let $f_{i}(x)$ be a monic polynomial over $R$ which has $a_{i}$ as a root. Let $R^{\prime}$ be the subring of $R$ generated by all the coefficients of $f_{1}(x), \ldots, f_{n}(x)$ over $B$. Then $R$ is a submodule of the finite $R^{\prime}$ module $A$, whence $R$ is a finite $R^{\prime}$-module, and $R$ is finitely generated over $B$.

Now we are to prove our main theorem:

TheOREm 3. Let $B$ be a pseudo-geometric ring and let $A$ be a B-algebra of finite type. Let $R$ be $B$-subalgebra of $A$. If $R$ is strongly submersive in $A$, then $R$ modulo its radical is a B-algebra of finite type.

The first step. We may assume that the radical of $A$ is zero, because of the assumption and the assertion. The condition of strong submersiveness is maintained even if $A$ is replaced by an $A^{\prime}$ which is a finite $A$-module and which is a subring of the total quotient ring of $A$. Therefore, first, we may assume that $A$ is the direct sum of a finite number of integral domains $A_{i}(i=1, \ldots, s)$ which are $B$-algebras of finite type. Then, since $A_{i}$ are pseudo-geometric, we may assume furthermore that each $A_{i}$ is a normal ring. Let $\mathfrak{a}_{i}$ be the kernel of the natural homomorphism from $R$ into $A_{i}$. If height $a_{\alpha}>0$ for an $\alpha$, then the direct summand $A_{\alpha}$ may be omitted because $A_{\alpha}$ has no effect for the validity of strong submersiveness.

The second step. Let $Q$ be the total quotient ring of $R$. Then by the assumption made above, $Q$ is a subring of the total quotient ring of $A$. Hence we can consider $R^{\prime}=A \cap Q$. Then the strong submersiveness of $R$ in $A$ implies that $R$ is strongly submersive in $R^{\prime}$. Since $R^{\prime}$ is contained in $Q$, we see that $R^{\prime}$ is integral over $R$. Hence, by virtue of Lemma 3.1, it is enough to show that $R^{\prime}$ is a $B$-algebra of finite type. Since $R^{\prime}$ is strongly snbmersive in $A$, we may assume that $R=R^{\prime}$. Then $R^{\prime}$ is the direct sum of a finite number of 
normal rings, say $R_{1}, \ldots, R_{t}$. Let $e_{i}$ be the identity of $R_{i}$. Then $R_{i}$ is strongly submersive in $A e_{i} . \quad R$ is a $B$-algebra of finite type if and only if all the $R_{i}$ are $B$-algebras of finite type. Therefore we may assume that $R$ is a normal ring. $B$ may also be replaced by a $B$-algebra of finite type contained in $R$. Therefore we may assume that $B$ is a normal ring and that the field of quotients of $B$ is the field of quotients $Q$ of $R$. Thus we have assumed that:

(i) $R$ is a normal ring with field of quotients $Q$,

(ii) $A$ is the direct sum of normal rings $A_{1}, \ldots, A_{s}$; each $A_{i}$ is a $B$-algebra of finite type, and, denoting by $\varepsilon_{i}$ the identity of $\mathrm{A}_{i}$, the ring $R_{i}$ is naturally isomorphic to $R$.

(iii) $R=A \cap Q$,

(iv) $B$ is a subring of $R$, the field of quotients of $B$ is $Q$ and $B$ is a normal ring.

The third step. When $S_{i}$ is a subset of $A_{i}$, we denote by $\sigma\left(S_{i}\right)$ the set of elements $a$ of $A$ such that $a \varepsilon_{i} \in S_{i}$. An ideal $a$ of $A$ is a prime ideal if and only if there is a prime ideal $\mathfrak{p}_{i}$ of $A_{i}$ for a suitable $i$ such that $a=\sigma\left(\mathfrak{p}_{i}\right)$. We note also that with symbols as above, $\left(R \cap \sigma\left(S_{i}\right)\right)_{\varepsilon_{i}}=R \varepsilon_{i} \cap S_{i}$.

Lemma 3.2. $R$ is a Krull ring. For each prime ideal $p$ of height 1 in $R$, there is a prime ideal $\mathfrak{p}_{i}$ of height 1 in $A_{i}$ for a suitable $i$ such that $\mathfrak{p}=\sigma\left(p_{i}\right) \cap R$.

Proof. Since $R=Q \cap A$, we see that $R$ is the intersection of Noetherian valuation rings $Q \cap \sigma(V)$, where $V$ runs through $\left(A_{i}\right)_{\mathfrak{p}_{i}}$ for all possible $i$ and prime ideals $\mathfrak{p}_{i}$ of height 1 in $A_{i}$. Therefore we see the assertion easily.

Lemma 3.3. There is a normal affine ring $B^{\prime}$ over $B$ such that (1) $B^{\prime}$ is a subring of $R$ and (2) for every prime ideal $p$ of height 1 in $R, \mathfrak{p} \cap B^{\prime}$ is a prime ideal of height 1 in $B^{\prime}$.

Proof. For each affine ring $B^{\prime}$ over $B$ such that $B^{\prime} \subseteq R$, let $E\left(B^{\prime}\right)$ be the set of prime ideals $\mathfrak{p}$ of height 1 in $R$ such that $\mathfrak{p} \cap B^{\prime}$ is not of height 1 . Note that since $B^{\prime}$ and $R$ have $Q$ as common field of quotients, $\mathfrak{p} \cap B^{\prime}$ is different from 0 . We see obviously that if $B^{\prime \prime} \subseteq B^{\prime}$, then $E\left(B^{\prime}\right) \subseteq E\left(B^{\prime \prime}\right)$. Since $A_{i}$ is an affine ring over $B \varepsilon_{i}$, there is a pair of a non-zero elemet $b_{i}$ of $B$ and a transcendence base $z_{i 1}, \ldots, z_{i t(i)}$ of $A_{i}$ over $B_{i}$ such that $A_{i}\left[b_{i}^{-1} \varepsilon_{i}\right]$ is integral over $\varepsilon_{i} B\left[b_{i}^{-1}, z_{i 1}, \ldots, z_{i t(i)}\right]$. Therefore, if a prime ideal $\mathfrak{p}$ of height 1 in $R$ does not contain any of $a_{1}, \ldots, b_{s}$, then considering a prime ideal $p_{i}$ of $A_{i}$ such that 
$\mathfrak{p}=\sigma\left(\mathfrak{p}_{i}\right) \cap R$, we have that $\mathfrak{p}_{i} \cap B \varepsilon_{i}$ is of height 1 in $B \varepsilon_{i}$, whence $\mathfrak{p} \cap B$ is of height 1. This shows that $E(B)$ is a finite set. Therefore, we have only to show that if $\mathfrak{p} \in E(B)$, then there is an affine ring $B^{\prime}$ over $B$ such that $B^{\prime} \subseteq R$ and such that $\mathfrak{p} \notin E\left(B^{\prime}\right)$. Theorem 2 shows that $R_{\mathfrak{p}}$ is a locality over $B$, whence there is a finite number of elements $a_{1}, \ldots, a_{r}$ of $R$ such that $R_{\mathfrak{p}}$ is a ring of quotients of $B\left[a_{1} \ldots, a_{r}\right]$. Then $E\left(B\left[a_{1}, \ldots, a_{r}\right]\right)$ does not contain $\mathfrak{p}$ and the proof of Lemma 3.3 is completed.

Now, by virtue of the above lemma and the proof, we may assume that for every affine ring $B^{\prime}$ over $B$ such that $B^{\prime} \subseteq R$, it holds that $E\left(B^{\prime}\right)$ is empty.

Lemma 3.4. Let $F(B)$ be the set of prime ideals $\mathfrak{p}^{\prime}$ of height 1 in $B$ such that there is no prime ideal $\mathfrak{p}$ of height 1 in $R$ such that $\mathfrak{p} \cap B=\mathfrak{p}^{\prime}$. Then $F(B)$ is a finite set.

Proof. If $b_{1}, \ldots, b_{s}$ are taken as in the proof of Lemma 3.3, then we see that every member of $F(B)$ contains some of $b_{i}$ as is seen quite easily. Therefore the assertion is proved.

Now we have the following, by virtue of Lemma 2.4 .

Lemma 3.5. Let a be the intersection of the members of $F(B)$. Then, under the assuption made just before Lemma 3.4, we see that $R$ is the a-transform $T(a)$ of $B$.

The fourth step. We proceed with the proof of our main theorem by induction on the altitude ( = Krull dimension) of $B$. Let $x$ be a transcendental element over $B$. Then for any prime ideal $q$ of height at least 2 , there is a prime element $p$ of $B(x)$ such that $p \in q B(x)$. Lemma 2.6 shows that $B$ may be replaced by $B(x)$. We want to show that $R$ is Noetherian. Let $q$ be an arbitrary prime ideal of $R$. We have only to show that $q$ has a finite basis.

(1) When $q$ contains a prime element $p . R / p R$ is strongly submersive in $A / p A$; these are $B /(p R \cap B)$-algebras. Therefore by our induction assumption, $R / p R$ is finitely generated over $B /(p R \cap B)$. Therefore $q / p R$ has a finite basis, which shows that $q$ has a finite basis.

(2) When height $q \geq 2$. $q B(x)$ contains a prime element $p$ as remarked above. If $a \subseteq p B(x)$, then $R \otimes B(x)$ contains $p^{-1}$, which is not the case. Therefore $p$ remains to be prime in $R \otimes B(x)$ by Lemma 2.8 Therefore (1) above shows that $q(R \otimes B(x))$ has a finite bașis. Therefore $q$ has a finite basis, 
(3) When height $q=1$. Set $q^{\prime}=B \cap q$. Then $B_{q^{\prime}}=R_{q}$ by Lemma 2.3. Set $q^{\prime \prime}=q^{\prime} R: q . \quad q^{\prime \prime}$ is not contained in any prime ideal of height 1 . Therefore height $q^{\prime \prime} \geq 2$. As in (2) above, $q^{\prime \prime}(R \otimes B(x))$ contains a prime element, and therefore $q^{\prime \prime}$ has a finite basis. Then we see that $q^{\prime \prime} / q q^{\prime \prime}$ is a finite $R / q$-module. Since $R / q$ is Noetherian by (2) above, we see that $\left(q \cap q^{\prime \prime}\right) / q q^{\prime \prime}$ is a finite $R / q$ module. Therefore $q \cap q^{\prime \prime}$ is generated by a finite number of elements by virtue of the fact that $\mathfrak{q}^{\prime}$ has a finite basis. On the other hand, since $R / \mathfrak{q}$ and $R / \mathfrak{D}^{\prime \prime}$ are Noetherian, we see that $R /\left(q \cap q^{\prime \prime}\right)$ is Noetherian. This and that $q \cap q^{\prime \prime}$ has a finite basis imply that $q$ has a finite basis. Thus $R$ is Noetherian.

The fifth step. Assume for a moment that $R$ is not finitely generated over $B$. Then we have a sequence of rings $B=B_{0}, B_{1}, B_{2}, \ldots$ with ideals $a_{0}, a_{1}, a_{2}$, ... respectively, as in the proof of Lemma 2.7. Let $a^{*}$ be the union of all $a_{i}$ and let $\mathrm{m}^{*}$ be a minimal prime divisor of $a^{*}$ (in $R$ ). We may assume that $B=B_{\mathrm{m}}$ with $\mathrm{m}=\mathrm{m}^{*} \cap B$. Let $B^{*}$ be the completion of $B$. Then $R \otimes B^{*}$ is strongly submersive in $A \otimes B^{*}, R \otimes B^{*}=T\left(a B^{*}\right)$, and $R \otimes B^{*}$ is not finitely generated over $B^{*}$. Therefore we may replace $B$ with $B^{*}$, dropping the condition that $B$ is a normal ring but assuming that the radical of $B$ is zero. Then, repeating the steps as before, we may assume the conditions at the end of the fourth step and also that $B$ is of finitely generated type over a complete local ring. This assumption enables us to use altitude formula. In particular, we have that if we enlarge $B$ so that (i) $\mathrm{m}^{*}$ is generated by $m$ and (ii) $R / \mathrm{m}^{*}=B / \mathrm{m}$ (this is possible, because if $p$ is a prime element of $R$ contained in $\mathrm{m}^{*}$, then $R / p R$ is finitely generated over $B /(p R \cap B)$ by our induction assumption), then we see that height $\mathrm{m}^{*}=$ height $\mathrm{m}$. Now, we consider again the completion $B^{*}$ of $B$. Let $q^{*}$ be an arbitrary prime ideal of height 0 in $B^{*}$. Let $q^{* *}$ be the prime ideal of $R \otimes B^{*}$ which lies over $q^{*}$. Then $R \otimes B^{*} / q^{* *}$ is strongly submersive in $\left(A \otimes B^{*}\right) / q^{* *}\left(A \otimes B^{*}\right)$. Therefore the derived normal ring $\bar{R}\left(q^{* *}\right)$ of $\left(R \otimes B^{*}\right) / q^{* *}$ is a Krull ring, and if $p^{* *}$ is a prime ideal of height greater than 1 in the ring, then $\mathfrak{p}^{* *} \cap\left(R \otimes B^{*}\right) / \mathfrak{q}^{* * *}$ is of height greater than 1 because of the altitude formula. Therefore we see that $a \bar{R}^{(* *)}$ is of height greater than 1 . This shows by virtue of Lemma 2.9 that $\bar{R}\left(q^{* *}\right)=\left(T\left(q B\left(q^{*}\right)\right)\right.$, where $B\left(q^{*}\right)$ is the derived normal ring of $B / q^{*}$. If every $\bar{R}\left(q^{* *}\right)$ is finitely generated over $B / q^{*}$, then, because of the fact that $R \otimes B^{*}$ is a subring of the total quotient ring of $B^{*}$, we see that $R$ must be finitely generated over $B$ (see the 
second step). Thus we may assume that $B$ is a complete local integral domain with maximal ideal $\mathrm{m}$ and the $R / \mathrm{m} R$ is a finite $B / \mathrm{m}$-module. Then we see that $R_{\mathfrak{m} *}=B$ by $(37,4)$ in [7], this is a contradiction to our choice of $\mathrm{m}^{*}$. Thus $R$ must be finitely generated over $B$.

\section{BibLIOGRAPHY}

[1] A. Grothendieck, Eléments de géométrie algébrique, Publ. Math. Inst. Etudes Sci. No. 4 (1960).

[2] - Seminaire de géométrie algébrique 1961, Inst. Haut. Études Sci.

[ 3 ] M. Nagata, A treatise on the 14-th problem of Hilbert, Mem. Coll. Sci. Univ. Kyoto, Ser. A Math. 30 (1956-57), 57-70. Addition and corrections, ibid. 197-200.

[4] - On the fourteenth problem of Hilbert, Proc. Internat. Congress of Math. 1958, Cambridge Univ. Press (1960), 459-462.

[5] - On the fourteenth problem of Hilbert, Lecture notes (by Murthy) at Tata Inst. F. R., forthcoming.

[6] - Invariants of a group in an affine ring, J. Math. Kyoto Univ. 3 (1963-64), 369377.

[ 7 ] - Local rings, Intersci. Tracts 13, John Wiley (1962).

[ 8 ] M. Nagata, T. Miyata, Remarks on matric groups, J. Math. Kyoto Univ. 4 (1964-65), 381-384.

[ 9 ] H. Weyl, Classical groups, Princeton Univ. Press (1939).

[10] O. Zariski, Interprétations algébrico-géométriques du quatorziéme problème de Hilbert, Bull. Sci. Math. 78 (1954), 155-168.

\section{Kyoto University}

